\title{
PHLPP1 PHOSPHATASE INHIBITION IN HYPOTHALAMUS RESTORES INSULIN SIGNALING AND ACTION AND REDUCES ADIPOSITY IN OBESE RATS
}

Bruna dos Santos Cardoso (IC), Gisele Castro (PG), Natália Ferreira Mendes (PG), Paula G. F. Quaresma $(P G)$, Tamires Marques Zanotto (PG), Mario J. Abdalla Saad (PQ), Patrícia de Oliveira Prada (PQ).

Abstract

PH Domain and Leucine Rich Repeat Protein Phosphatase 1 (PHLPP1) regulates the Akt activity in hypothalamus of adult rats. Obesity is responsable by the increase of PHLPP1 in the hypothalamus, inhibiting Akt activity, impairing the insulin action in the hypothalamus, resulting in insulin resistance. Thus, the silencing of this phosphatase could act in the regulation of Akt activity.

Key words: PHLPP1, Obesity, Hypothalamus.

\section{Introduction}

Obesity results from imbalance between food intake and energy expenditure. The energy homeostasis is regulated by hypothalamic neurons that receive different neural, hormonal and metabolic signals. Insulin is one of the main hormones that regulate energy homeostasis and acts through a cascade of intracellular signaling that depends on the activation of several proteins, such as Akt. Our hypothesis is that PHLPP1 inhibits Akt activity by dephosphorylating serine 473 residues, impairing the insulin action in the hypothalamus ${ }^{1 ; 2}$.

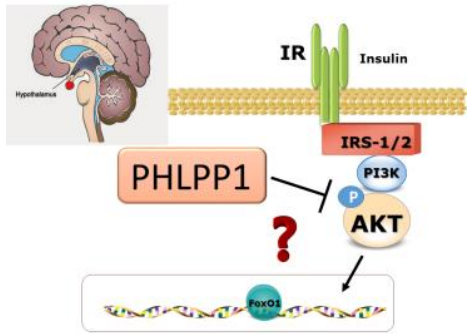

The objectives are investigate PHLPP1 protein expression in the hypothalamus of diet induced obesity (DIO) rats and to assess whether the PHLPP1 silencing improves insulin action and decreases body adiposity

\section{Results and Discussion}

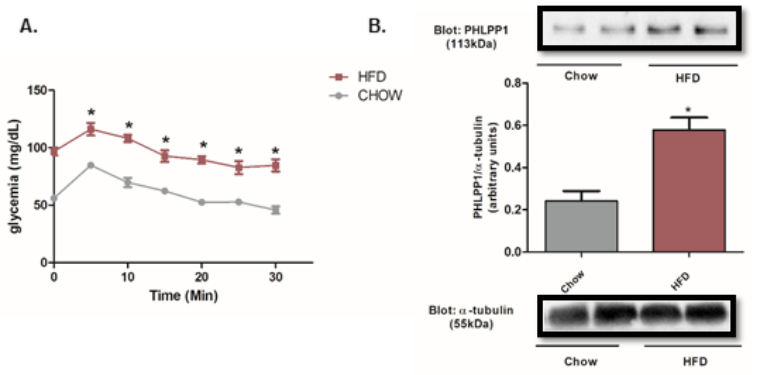

Image 1: Diet induded obesity

A. Intolerance Insulin Test (ITT), standard chow (Chow) and high fat diet

"
A.

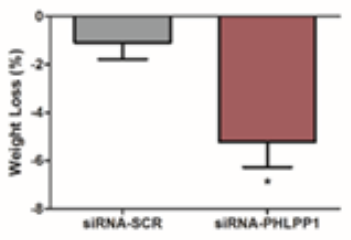

C.

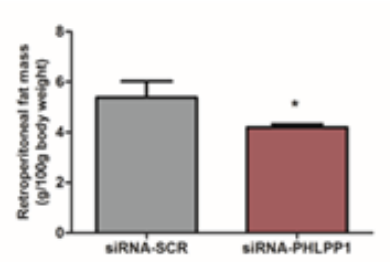

B.

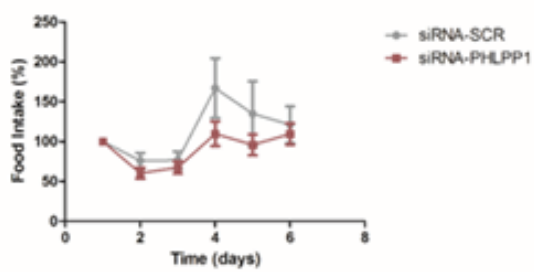

D.

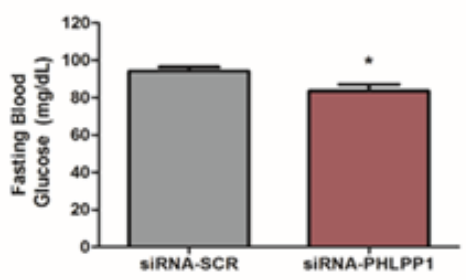

Image 2 Treatment to silence PHLPP1 expression.

A: Percent of weight loss after the treatament with siRNA. " $p=0,0098$. B: Food Intake. C: Mass of retroperitoneal adipose tissue. $" p=0,0497$. D: Fasting blood glucose after treatment with siRNA. " $p=0,0359$.

\section{Conclusions}

Hypothalamic PHLPP1 silencing in DIO animals restored, at least in part, the insulin signaling, promoting reduction in body weight and adiposity.

\section{Acknowledgement}

Sponsor Institutuion: PIBIC/Cnpq

${ }^{1} \mathrm{CHEN}$, Bo et al. PHLPP1 gene deletion protects the brain from ischemic injury. Journal of Cerebral Blood Flow \& Metabolism, v. 33, n. 2, p. 196-204, 2013.

${ }^{2}$ NEWTON, Alexandra C.; TROTMAN, Lloyd C. Turning off AKT: PHLPP as a drug target. Annual review of pharmacology and toxicology, v. 54, p. 537, 2014. 